\title{
Knowledge Management: A Neuro-Hemispherical view of the field
}

\author{
Amir M. Sharif ${ }^{1}$ \\ Information Systems Evaluation and Integration Group (ISEing) \\ School of Information Systems, Computing and Mathematics \\ Brunel University \\ Uxbridge, UB8 3PH
}

\begin{abstract}
The definition and subsequent use of knowledge within and across organisational and social contexts has been a vibrant and evolving growth area over many years. Understanding the notion of Knowledge Management (KM) as an ensemble approach, through the codification, manipulation, dissemination and distribution of information, poses more questions than it answers. The ability to recognise the basis of KM in this regard, involves the tracing of a social or a systems view of knowledge, across cultural contexts (most notably in terms of Western or Eastern philosophies and ontologies). By highlighting the weak and strong push / pull forces of codification versus collaboration in such a manner, can provide us with a possible technique to discern between these worldviews of knowledge and thus ameliorate the many definitions of $\mathrm{KM}$ and the associated complexity of its implementation.
\end{abstract}

Keywords: Knowledge Management, Cross-cultural context, Cognitive lens

\footnotetext{
${ }^{1}$ Visiting Research Fellow, email: ams@amir.demon.co.uk
} 
Providing reasons and explanations for something you don't understand or know about, is, on a philosophical level, one of the most fundamentally difficult and complex tasks that humans are confronted with. This difficulty arises as a direct result of trying to compare and realise the unknown with the known. And so it is the case with the definition of knowledge. How do we 'know' what knowledge is? How can we 'manage' it, let alone define it, if we are still unsure of its constituent existence? There are numerous books, articles and periodicals spread across fields as diverse as business, psychology and Information Systems (IS), that are focussed on this area (be it in terms of philosophical, methodological or implementation issues). However, a plethora of definitions and points of view (such as this), exist seeking to quantify knowledge. Try asking 20 people chosen at random to define knowledge, and you might get back 20 different answers (maybe even more if they think about it for a while). What you may well find is that we tend to sense or think that knowledge is a complete tautological entity in itself: it just is, what it is, because it is. This self-affirmation is unique in that no further proof or existence of supporting evidence is required in order for us to think about knowledge in any other terms.

Knowledge is structured, yet amorphous, meta-information: the essential sublimation and distillation of experiential evidences, confirmatory / negatory results and associated self evident truths. Yet how is this made manifest? Principally, through the lenses of culture and socio-psychological interactions. Certainly, with regards to knowledge management $(\mathrm{KM})$, the growth and interest in this field has principally been due to business and management thinking on both sides of the Pacific Ocean. This encompasses thinking about knowledge in individual, team, organisational or societal terms.

It is for this reason that I believe that a Neuro-Hemispherical divergence or a geographically-influenced cognitive model of knowledge exists in this field. In other words, a cultural-driven lens on knowledge in terms of both Eastern and Western frames-of-reference compete for attention in this space. Are we allied to the explicittacit (Socialisation, Externalisation, Collaboration, Internalisation) world of Nonaka and Takeuchi (1995) or the systematic, codification processes as highlighted by Davenport and others (Davenport and Prusack, 1998; Sveiby, 1997; Wiig, 1997)? Or should we, 
in my opinion, and as also opined recently by Zhu (2004), take on board the best aspects of both? Indeed as Zhu notes, the complexity of understanding how best to understand and manage knowledge is further broken down by country differences in each geographic area: being American, Japanese, Chinese and European views respectively. As such, this makes understanding knowledge and placing it within the proper context more difficult because of the particular cultural and systems thinking influences that drive each form of KM. At the same time as freeing us to think along those lines, we are in a sense, inhibited (at best) and / or restricted (at worst) by the cultural boundaries of each frame of reference: be it systems thinking led or human capital-led. These two "Neuro-Hemispherical" worldviews as I will call them, clearly represent a schism within the $\mathrm{KM}$ literature, being based upon either Codification or Collaboration concepts.

In the former, largely Western-view of KM, there is a strong emphasis on implementing processes and systems that capture and store knowledge from individuals. Here, the benefits of eliciting both primary and secondary sources of knowledge are largely seen as having immediate or at least short-term impact. By codifying and providing information taxonomies, within organisational KM systems say, the means for accessing and making use of knowledge is achieved in a 'pull' sense. In other words, it is up to the seeker of knowledge to seek and find his/her relevant information, via search and data mining tools. In the latter Eastern view of $\mathrm{KM}$, there is conversely a strong emphasis on providing means for individuals and teams with the ability to share knowledge via the concept of communities, within which the sharing or collaboration of knowledge is encouraged, i.e. in a 'push' sense. This engenders a long-term approach to the realization of benefits that such a people-focussed view affords.

At the same time, there is a weaker supporting reliance upon Collaborative knowledge also, from the point of view of the Western outlook on KM. That is to say that there is a greater tendency to apply a systems-thinking lens to knowledge. Conversely, the Eastern view of $\mathrm{KM}$ has a weak reliance upon the more structured systems view. Again, this is largely due to a reliance and dependency on individuals and teams to collaborate in order to share knowledge. This is shown in Figure 1. 


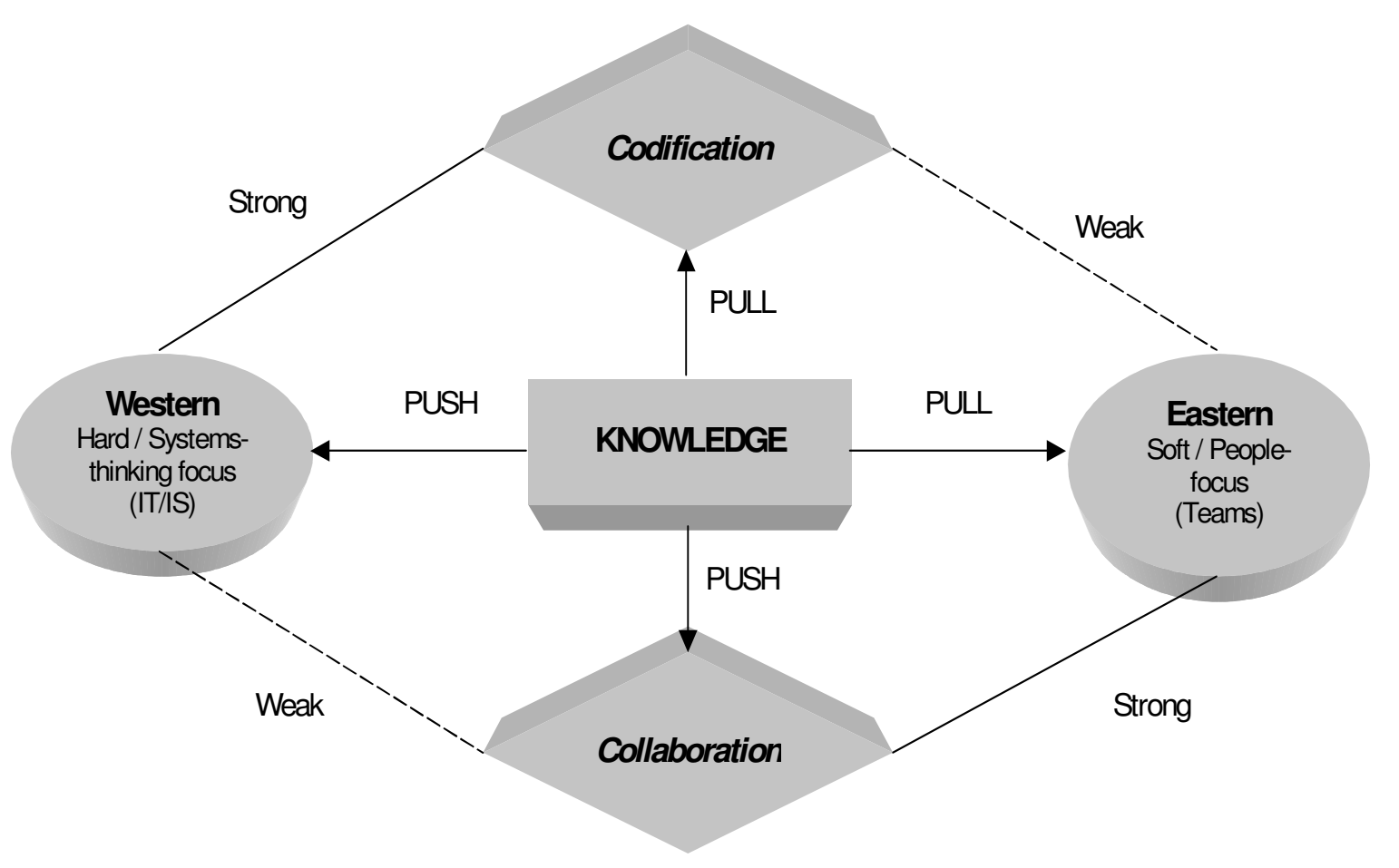


Currently, the field of KM therefore appears to me to be a conglomeration of either Asian or Americo-European views, which needs to be borne in mind when considering each set of cognitive models of knowledge management. As far as I have seen, there has been little work on extending these ideas, in the normative literature. However, having noted these details, the application of each subsequent lens on $\mathrm{KM}$, has been successfully applied and transplanted from one cultural context to the other. For example Davenport and Prusack (1998), there are numerous accounts of multinational organizations trying to apply a combination of techniques in order to make the best use of their knowledge workforce. What is therefore required is a technique to discern or even amalgamate these approaches - as such a number of research questions need to be addressed:

- Which hemispherical lens do you wish to view knowledge in (KM in the large)?

- In what circumstances and for how long is such a view valid?

- Who is impacted by this knowledge?

- What can be done to manage these factors (KM in the small)?

In doing so, a better appreciation of what constitutes knowledge and how it can potentially be managed can be put into cultural and / or cross-cultural contexts, as required.

\section{Disclaimer}

The author wishes to assert that the views expressed in this article are solely that of the author.

\section{References}

Davenport TH and Prusack L (1998) Working Knowledge: How Organizations Manage What They Know. Harvard Business School Press, Boston.

Nonaka I and Takeuchi H (1995). The Knowledge-creating Company: How Japanese Companies Create the Dynamics of Innovation. Oxford University Press, Oxford. Sveiby KE (1997) The New Organisational Wealth. Berret-Koehler, San Francisco. Wiig KM (1997). Knowledge Management : An Introduction and Perspective. Journal of Knowledge Management, 1 (1), 6-14.

Zhu Z (2004) Knowledge Management: towards a universal concept or cross-cultural contexts? Knowledge Management Research and Practice, 2 (2), 67-79. 
Figure 1. A neuro-hemispherical view of Knowledge 